RHIC-PH-15

Review of High Energy Hadron-Nucleus Data

D. Lissauer

Brookhaven National Laboratory

'August 1986 


\title{
REVIEW OF HIGH ENERGY HADRON-NUCLEUS DATA
}

\author{
D. Lissauer
}

Physics Department

Brookhaven National Laboratory, Upton, New York 11973

and

Physics Department

Tel-Aviv University, Israel

Presented at the 5th International Conference on U1tra-Relativistic Nucleus-Nucleus Collisions (Quark Matter '86)

Asilomar - Pacific Grove, California April 13-17, 1986 
D. Lissauer

Physics Department, Brookhaven National Laboratory, New York Physics Department, Te1-Aviv University, Israel

In this review we will summarize new data on hadron-nucleus interactions. The possibility that quark-gluon plasma may be created in heavy ion collisions has led to renewed interest in hadron-nucleus collisions. In particular one hopes that understanding the energy loss of hadrons in h-A collisions will allow us to estimate the optimum energy in AA collisions in order to achieve maximum baryon and/or maximum energy density. This will allow us to choose the optimal experimental environment in the search for quark-gluon plasma. This review will thus omit many interesting results from hadron-nucleus collisions, such as the A dependence of lepton pair production, EMC effect and others. We will focus our attention on the following:

(i) Estimating the rate of energy loss of the incident hadron as it propagates through the target.

(ii) Determining where the energy is deposited in central hadronnucleus collisions.

It is clear that there is no direct or unique method of extrapolating our knowledge of $\mathrm{h}-\mathrm{A}$ collisions to predict what will happen in AA-collisions. The knowledge and understanding of $\mathrm{pP}$ and $\mathrm{pA}$ collisions is, however, a useful and necessary guide to what one can expect in AA collisions.

In this review we will concentrate on three experimental approaches to the study of $\mathrm{h}-\mathrm{A}$ collisions. In Section 1 we will discuss the present status of $\mathrm{pA} \rightarrow \mathrm{p}+\mathrm{X}$ inclusive measurements. In Section 2 measurements from visual detectors, in this case results from the $30^{\prime \prime}$ hybrid spectrometer, which allows investigations of global event properties will be presented. In Section 3 data using $2 \pi$ calorimeters, where one can trigger and measure transverse energy and energy flow over a given rapidity region, will be discussed. The conclusions will be given in Section 4.

\section{INCLUSIVE PROTON SPECTRA FROM P-A INTERACTIONS}

Nuclear stopping power can be thought of as a rate of energy. loss of a hadron traversing nuclear matter. One of the first analyses of nuclear stopping power using inclusive proton measurement at fixed $\mathrm{p}_{\mathrm{T}}$ was performed by Busza and Goldhaber. ${ }^{1}$ The above analysis used the data of Barton et a1. ${ }^{2}$ who measured inclusive $\mathrm{p}$ spectra in a pA collisions at $100 \mathrm{Gev} / \mathrm{c}$ for different targets at fixed $P_{T}, P_{T}=0.3 \mathrm{Gev} / \mathrm{c}$ and $0.5 \mathrm{Gev} / \mathrm{c}$. The data is summarized in Figure 1. Using these results, the differential probability density $\rho(\Delta y)(\Delta y \cong 1 \mathrm{nx})$ was calculated for $\mathrm{pP}$ and $\mathrm{p} \mathrm{Pb}$. In addition we show the calculated $\rho(\Delta y)$ for $P \mathrm{~Pb}$, peripheral, averge and central colli*Work has been performed under the auspices of the U.S. Department of Energy under Contract No. DE-AC02-76CH00016. 
Figure 1. Inclusive proton cross section $E \mathrm{~d} \sigma / \mathrm{d}^{3} \mathrm{p}$ at $\mathrm{PT}_{\mathrm{T}}=0.3$ $\mathrm{Gev} / \mathrm{c}$, as a function of $\mathrm{X}$ for $\mathrm{pA} \rightarrow \mathrm{pX}$, at $100 \mathrm{Gev} / \mathrm{c}($ ref. 2).

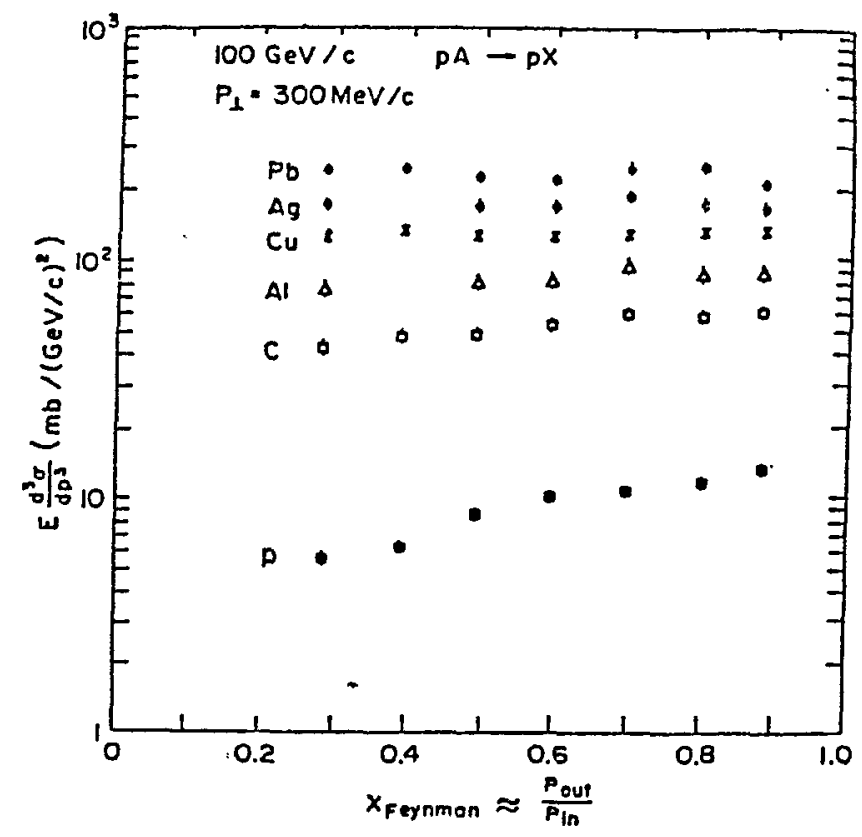

sions as calculated in reference 1 . The uncertainty in the estimated probabilities resulted from systematic error in the data as well as those introduced by the assumption. ${ }^{1}$ The above data has stimulated a large number of further investigations into the question of nuclear stopping power. ${ }^{3-6}$

At that time the only additional complete set of data available was at 24 Gev/c from T. Eichten et a1. ${ }^{7}$ Two additional sets of data became available only recently, one at $17 \mathrm{Gev} / \mathrm{c}$ from the AGS experiment of $\mathrm{L}$. Resmoberg et al. ${ }^{8}$ and the other of $\mathrm{R}$. Baily et $a 1 .{ }^{6}$ which used a $100 \mathrm{Gev} / \mathrm{c}$ beam from the SPS. In figure 3 we show the inclusive cross section at $p_{T}=0.3 \mathrm{Gev} / \mathrm{c}$ from the data at $100 \mathrm{Gev} / \mathrm{c}^{2} 17 \mathrm{Gev} / \mathrm{c}^{8}$ and $24 \mathrm{Gev} / \mathrm{c} .^{7}$ It is clear that the data are not consistent with each other. The fact that the slopes of the 100 Gev/c data and the lower energy data are different does not have to surprise us, since one does not expect Feyman scaling to hold down to lower energies. But when one compares the 24 and $17 \mathrm{Gev} / \mathrm{c}$ data sets we find that the slope is in reasonable agreement; on the other hand the absolute normalization seems to be different by about a factor of two. This disagreement is fundamental to our intepretation of the results. If only the $24 \mathrm{Gev} / \mathrm{c}$ and the $100 \mathrm{Gev} / \mathrm{c}$ data are compared, the conclusion would be that stopping power at $100 \mathrm{Gev} / \mathrm{c}$ is greater than that at $24 \mathrm{Gev} / \mathrm{c}$. If, however, we compare the 17 $\mathrm{Gev} / \mathrm{c}$ data to the $100 \mathrm{Gev} / \dot{c}$ and take that normalization to be correct, we will conclude that the stopping power at $17 \mathrm{Gev} / \mathrm{c}$ is 1 arger than at 100 Gev/c. This conclusion is based on the assumption that the proton-inclusive cross section has to be the same at 100 and $17 \mathrm{Gev} / \mathrm{c}$. The missing part of the cross section we thus assume has been stopped further and shifted to 
Figure 2. Probability distribution for protons to loose rapidity $-\Delta y$ in collisions of $\mathrm{PPb}$. Data from ref. 2 and

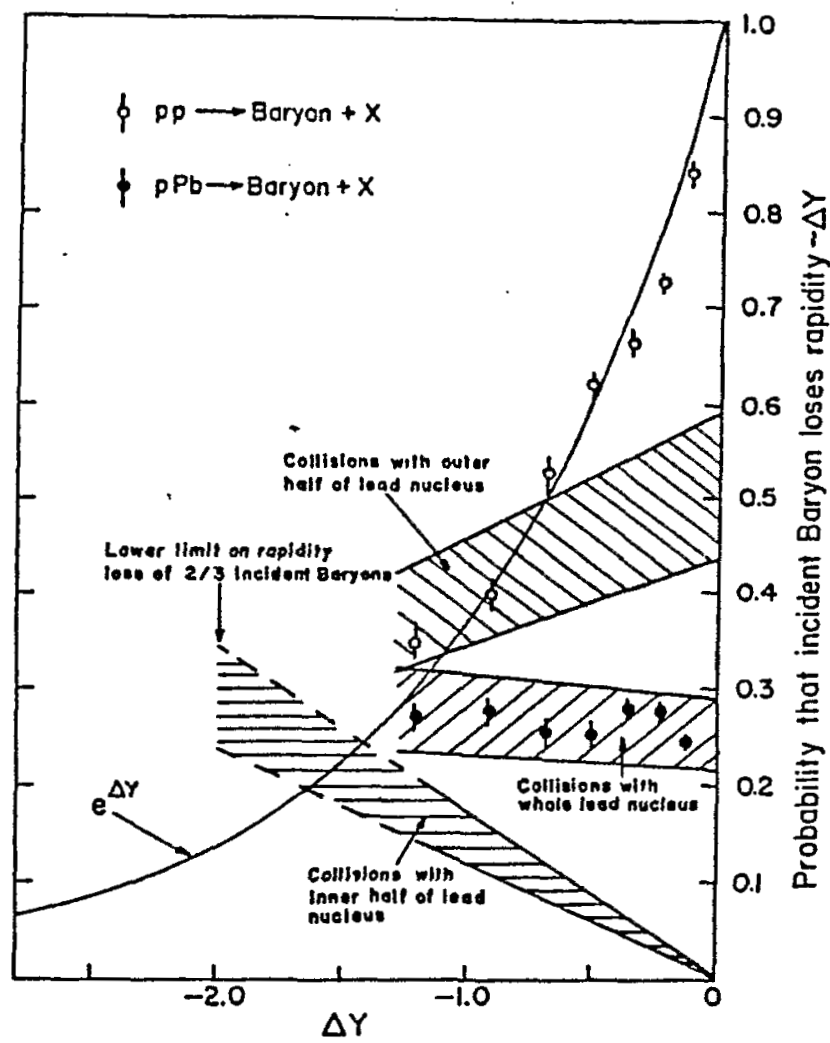
calculations from ref. 4.

lower $X_{F}$, where the experiment has no acceptance. ${ }^{8}$ This implies a stronger stopping at $17 \mathrm{Gev} / \mathrm{c}$. Such a conclusion obviously would have a favorable implication for the formation of high baryon density matter with heavy ion beams at AGS energies.

A comparison of the new SPS data ${ }^{6}$ with the Barton et al. data ${ }^{2}$ and its analysis by Buza and Goldhaber ${ }^{1}$ shows some discrepancy between the two experiments. The new data have measurements to lower $\mathrm{X}_{F}$ values, and show that the nucleon stopping power in nuclear matter is less than previous estimates.

The data we thus have at present is quite contradictory. While it is clear that measurement of $\mathrm{pA} \rightarrow \mathrm{P}+\mathrm{X}$ inclusive cross sections are of fundamental importance to our understanding of h-A collisions, or more specifically to the stopping mechanism, the unfortunate contradiction between different data sets does not allow unambiguous conclusions. New and precise measurements at different beam energies and different $A$ with preferably the same experimental set up are urgently needed. 


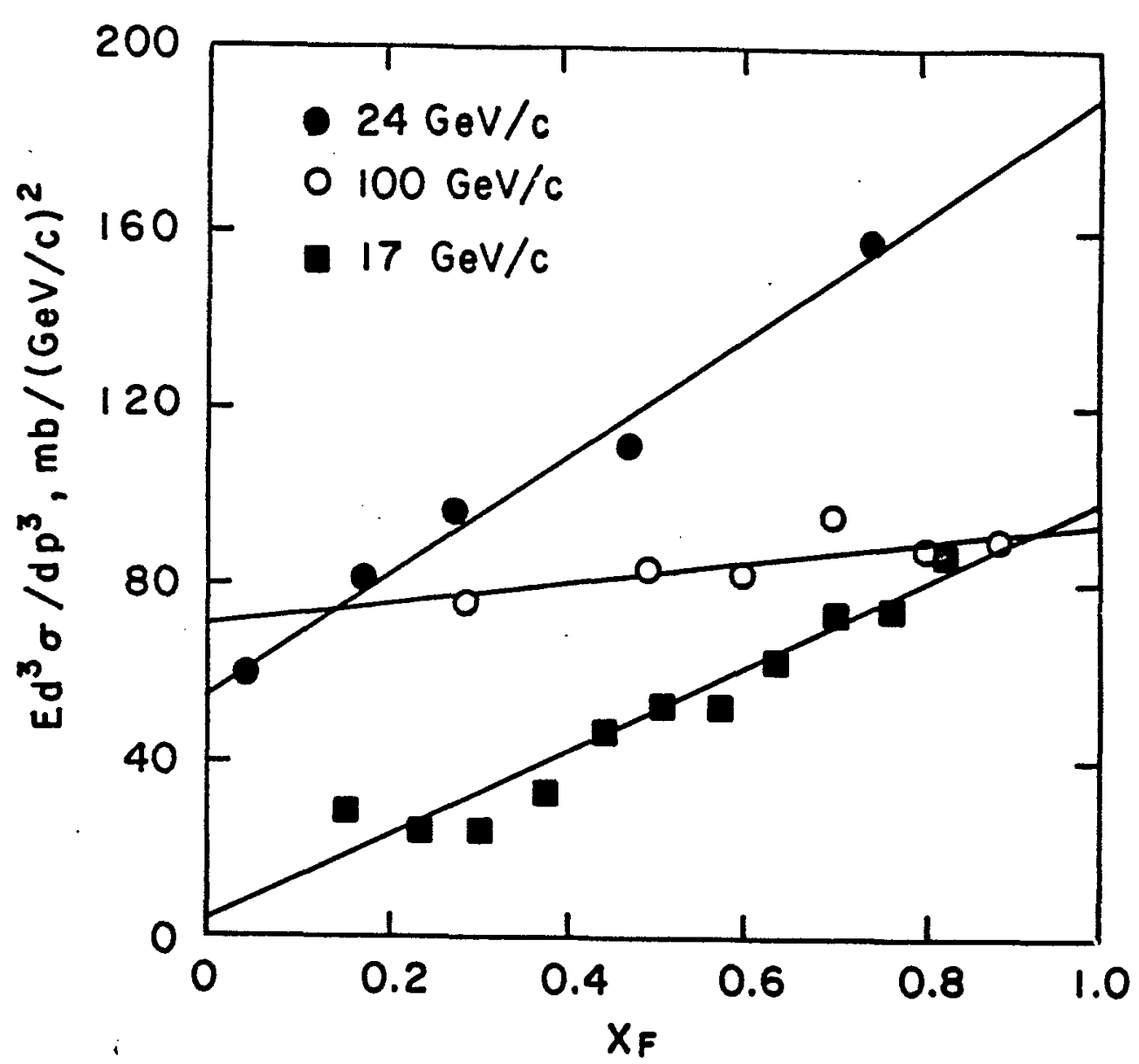

Figure 3. Inclusive proton cross section at $P_{T}=0.3 \mathrm{Gev} / \mathrm{c}$ for data: 17 $\mathrm{Gev} / \mathrm{c}^{8} 24 \mathrm{Gev} / \mathrm{c}^{7}$ and $100 \mathrm{Gev} / \mathrm{c}^{2}$

2. GENERAL PROPERTIES OF h-A COLIISIONS

Visual detectors such as emulsions, streamer chambers and bubble chambers have allowed the study of global properties of hA collisions. There are two new experiments from FNAL using the 30 " bubble chamber with an external spectrometer. The experimental setup is similar in both experiments. The Fermilab 30" hydrogen bubble chamber was fitted with three pairs of thin foils of silver, gold and magnesium. The bubble chamber allows a good $\pi / p$ discrimination using $\mathrm{dE} / \mathrm{dX}$ up to momenta of about $1 \mathrm{Gev} / \mathrm{c}$. The chamber was exposed to $\pi^{+}, \pi^{-}, P$ and $\bar{P}$ beams at energies of $100 \mathrm{Gev} / \mathrm{c}$ in W. D. Walker et $\mathrm{al} .{ }^{9}$ and $200 \mathrm{Gev} / \mathrm{c}$ for R. J. Ledoux et a1. 10

In the analysis of Walker et al. ${ }^{9}$ the events were divided according to their impact parameter and classified using the number of identified protons in the bubble chamber. The assumption is that the number of slow protons is indicative of the volume of nuclear matter in which the reaction takes place. On the average this is closely related to the chord length of the 
axis of the shower in the nuclear matter. In the analysis the events were separated into two groups. Peripheral interactions were defined as those having $N_{p} \leq 4$, and central collisions as those having $N_{p} \geq 5 . \quad\left(N_{p}=\right.$ number of slow protons.) This separation is statistical in nature and classifies about equal numbers of events as central and peripheral. In figure 4 we show the frequency spectrum of the number of identified protons. The curve is a prediction of a simple model which assumes that all nucleons in a volume of a truncated core of radius 1 fermi and half angle of 0.3 radians participate in the interaction.

In figure 5 we show the normalized multiplicity distribution for negative particles using the above event selection. The agreement with KNO scaling is reasonably good for the central collisions. This is especially true if one remembers that the average $\pi^{-}$multiplicity $<n>$, for these central colli-

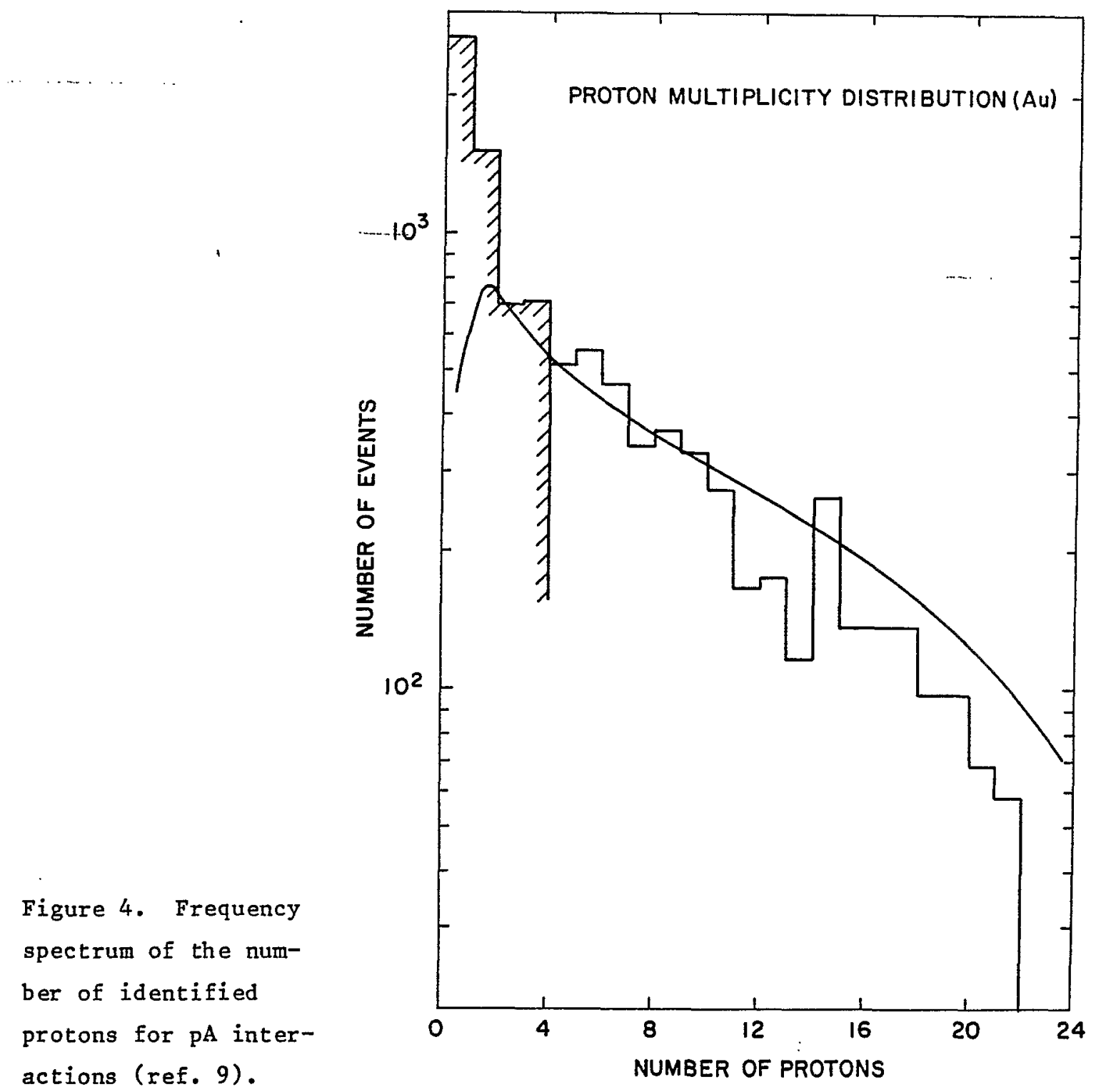




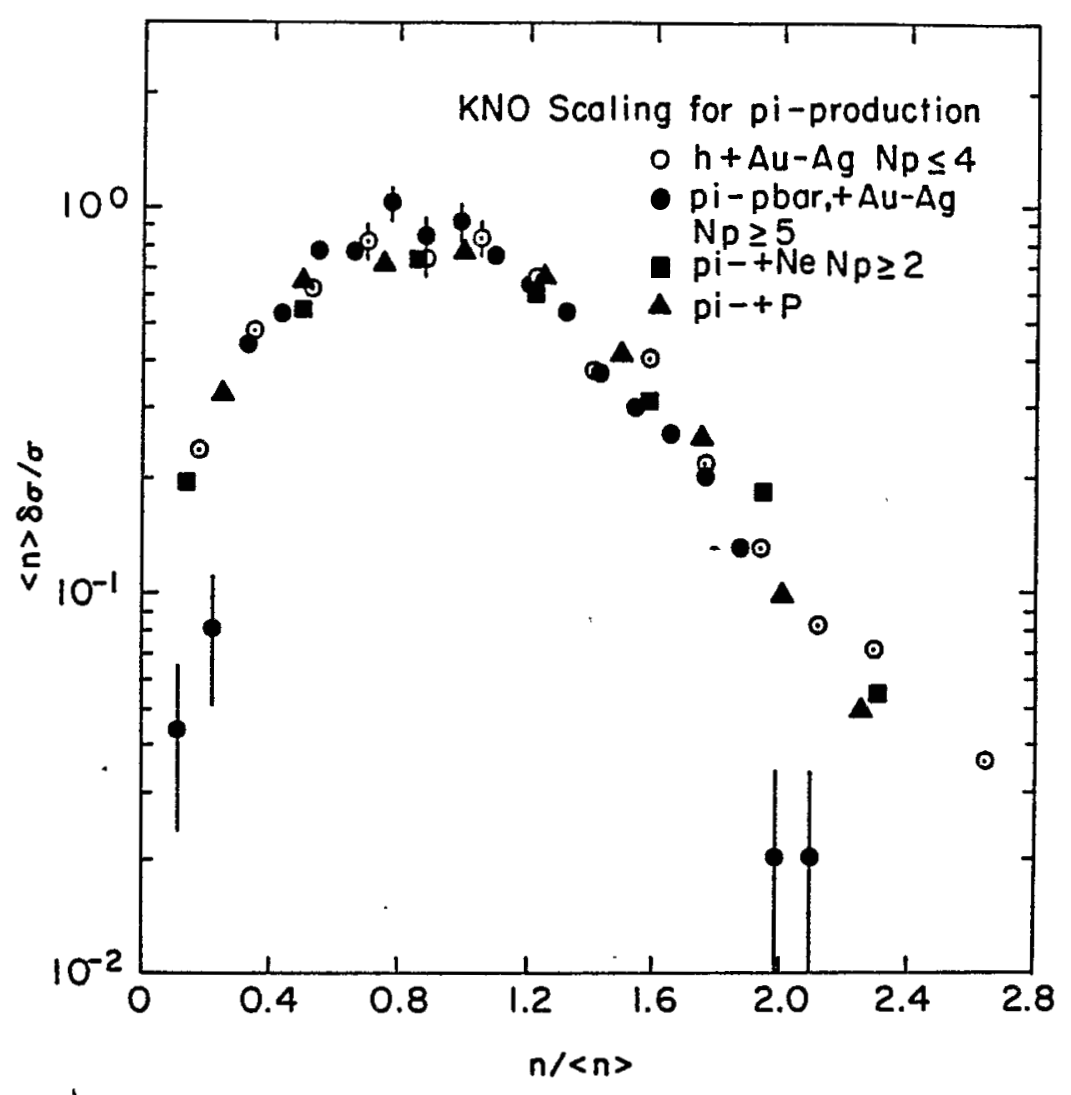

Figure 5. The multiplicity distribution $n /<n>$ for $\pi^{-}$production for different central and peripheral pA collisions (ref. 9).

sions is four times that for PP collisions. The deficit at small $n /\langle n>$ for central hA collisions is attributed to the lack of diffractive events. In figure 6 and figure 7 we show the rapidity distribution for different classes of events. A clear attenuation of the leading particle is observed as well as an enhancement at low rapidity starting at about 3.5 units of rapidity.

In the analysis of reference 9 an attempt was made to measure directly the amount of energy flow between the forward, central and backward region for the peripheral and central collision events. In Table 1 we show their results for $\pi^{+}\left(\pi^{-}\right)$A interaction. The forward region is defined to be for particles with momenta greater than $20 \mathrm{Gev} / \mathrm{c}$, the central region for particles between 10 to $20 \mathrm{Gev} / \mathrm{c}$, and the backward region for particle with $\mathrm{P} \leq$ $100 \mathrm{Gev} / \mathrm{c}$. The table shows the average number of particles as well as the average momenta carried by particles in these regions for $\underline{p}$ light ion, peripheral pA and central pA collisions. The most striking feature in the table is a clear reduction in the number of leading particles for central 


$$
\begin{aligned}
\pi^{+}\left(\pi^{-}\right) & \text {Collisions } \\
\text { P } & >20 \mathrm{Gev} / \mathrm{c}
\end{aligned}
$$

$\frac{\mathrm{N}_{\mathrm{p}}>1}{\pi^{+}\left(\pi^{-}\right)-\mathrm{P} \quad \pi^{+}\left(\pi^{-}\right)-\mathrm{Mg}} \frac{\mathrm{N}_{\mathrm{p}}<4}{\pi^{+}\left(\pi^{-}\right)-\mathrm{Ag} \quad \pi^{+}\left(\pi^{-}\right)-\mathrm{Au}} \frac{\mathrm{N}_{\mathrm{p}}>5}{\pi^{+}\left(\pi^{-}\right)-\mathrm{Ag} \quad \pi^{+}\left(\pi^{-}\right)-\mathrm{Au}}$

$\begin{array}{ccccccl}\mathrm{N}+(-) & .59(.49) & .59(.46) & .59(.53) & .57(.50) & .31(.25) & .26(.30) \\ \mathrm{N}-(+) & .20(.21) & .18(.29) & .23(.25) & .27(.25) & .19(.22) & .16(.21) \\ \mathrm{P}+(-) & 26.9(23.3) & 22.7(17.3) & 23.4(21.2) & 26.0(19.5) & 10.6(12.3) & 8.9(12.1) \\ \mathrm{P}-(+) & 7.3(7.1) & 6.8(10.2) & 9.0(9.6) & 10.1(9.5) & 7.5(7.6) & 7.2(6.5)\end{array}$

$10 \mathrm{Gev} / \mathrm{c} \leq \mathrm{P}_{\mathrm{I}} \leq 20 \mathrm{Gev} / \mathrm{c}$

$\begin{array}{lllllll}\mathrm{N}+(-) & .66(.33) & .56(.53) & .56(.55) & .51(.64) & .50(.40) & .56(.76) \\ \mathrm{N}-(+) & .27(.24) & .38(.48) & .30(.44) & .31(.37) & .38(.38) & .36(.53) \\ \mathrm{P}+(-) & 6.7(5.8) & 8.8(8.1) & 8.6(8.3) & 7.8(9.6) & 7.6(6.3) & 8.5(10.7)\end{array}$

\begin{tabular}{lcccccc}
\multicolumn{7}{c}{$\mathrm{P}_{\mathrm{L}} \leq 10 \mathrm{Gev} / \mathrm{c}$} \\
$\mathrm{N}+(-)$ & $3.0(2.5)$ & $4.3(4.2)$ & $4.3(4.0)$ & $6.2(3.7)$ & $8.2(7.6)$ & $9.2(7.6)$ \\
$\mathrm{N}-(+)$ & $1.9(2.6)$ & $3.7(6.0)$ & $3.4(4.1)$ & $3.2(4.8)$ & $6.2(8.6)$ & $7.3(8.7)$ \\
$\mathrm{P}+(-)$ & $5.6(5.6)$ & $11.6(10.6)$ & $10.5(10.0)$ & $11.3(9.3)$ & $16.2(16.6)$ & $17.7(13.6)$ \\
$\mathrm{P}-(+)$ & $3.8(5.3)$ & $8.6(9.1)$ & $8.6(9.6)$ & $7.8(10.0)$ & $10.6(16.5)$ & $12.3(16.1)$ \\
& & & & & \\
No. of Events & $92(62)$ & $230(174)$ & $173(128)$ & $108(85)$ & $96(86)$
\end{tabular}

collisions. This reduction is more pronounced for particles carrying the same charge as the beam. The total average momentum carried by all particles in the above momentum region is defined as $\underline{P}+(-)$ and $\underline{P}-(+)$. It is clear that there is a substantial loss of momenta carried by the leading particles when one moves from peripheral to central collisions. In the central region both the number of particles and the amount of momentum carried is relatively unchanged from the peripheral to central collisions. In. the backward direction we see a marked increase in the number of particles as well as in the total amount of momentum carried by the particles. Similar results are observed for $\mathrm{p} / \overline{\mathrm{p}}$ collisions.

From an estimate of the path length in nuclear matter one can calculate the average attenuation per fermi. In this analysis the authors ${ }^{9}$ have arrived at a measure of $\mathrm{dE} / \mathrm{dX}$ of about $3 \mathrm{Gev} /$ fermi that is transferred 


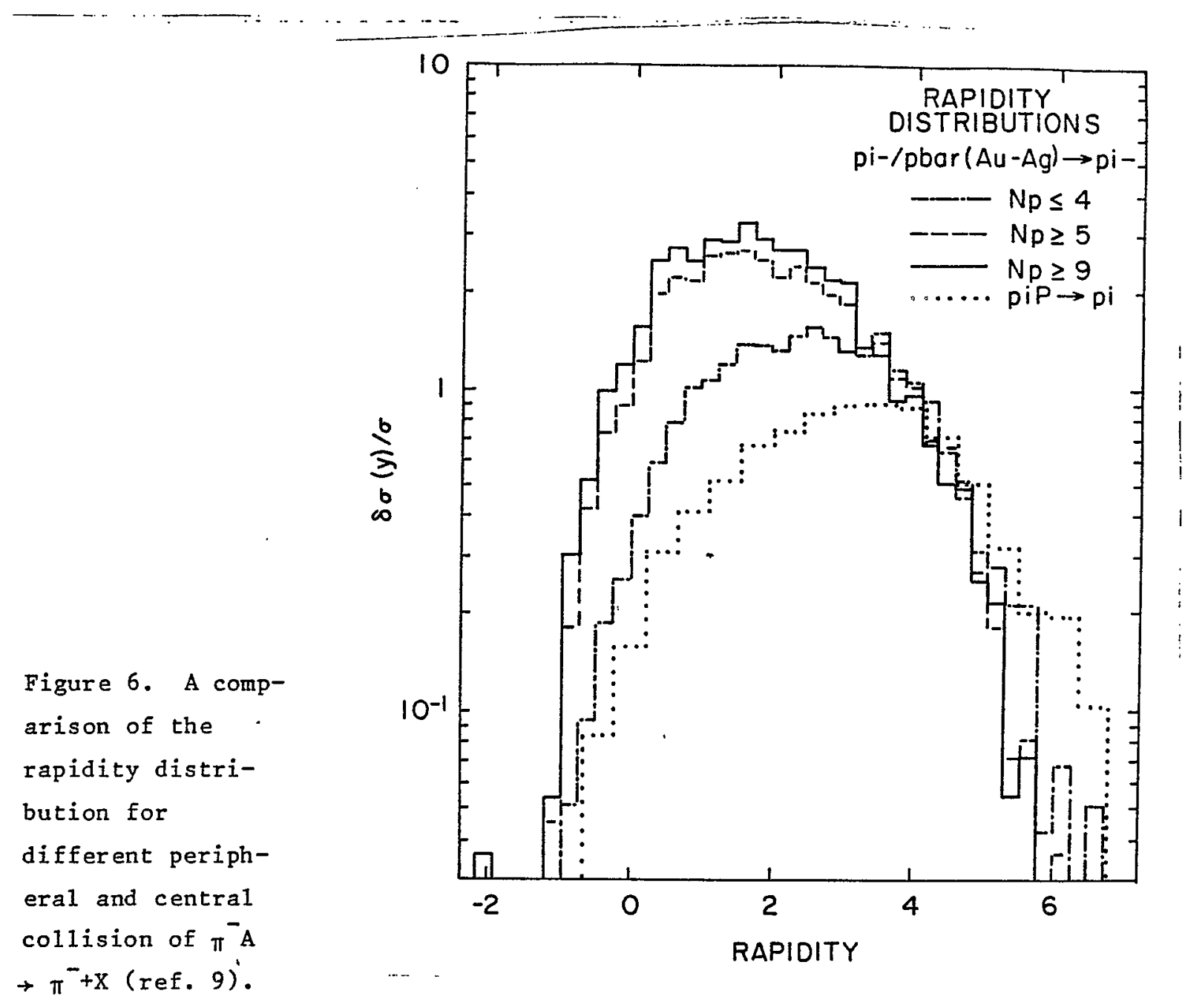

from the leading particles to the backward region. Note that this is an energy dependent variable.

The experiment of Ledoux et al ${ }^{10}$ used a beam momenta of $200 \mathrm{Gev} / \mathrm{c}$. In the analysis the authors have classified the events according to the number of interactions, $\nabla$, of the hadron in the nucleus as measured by the number of slow protons measured in the bubble chamber. They followed a formulation used previously ${ }^{11-12}$ where

$$
\mathrm{V}(\mathrm{np})=\mathrm{c}_{\mathrm{A}} \vee \mathrm{np}
$$

where $n p=$ number of slow protons with $P<1.5 \mathrm{Gev} / \mathrm{c}$

$\mathrm{C}_{\mathrm{A}}=$ constant for each target $\mathrm{A}$ such that $\langle\mathrm{r}\rangle$ determined via this, and that extracted from the inelastic cross section, agree. In figure 8 we show the mean $X=P / P_{\text {beam }}$ of the leading charge particle as a function of $\nu$. One sees a rapid decrease in the mean $X$ after the first few interactions; the rate of decrease slows as the number of interaction increases. In figure 9 


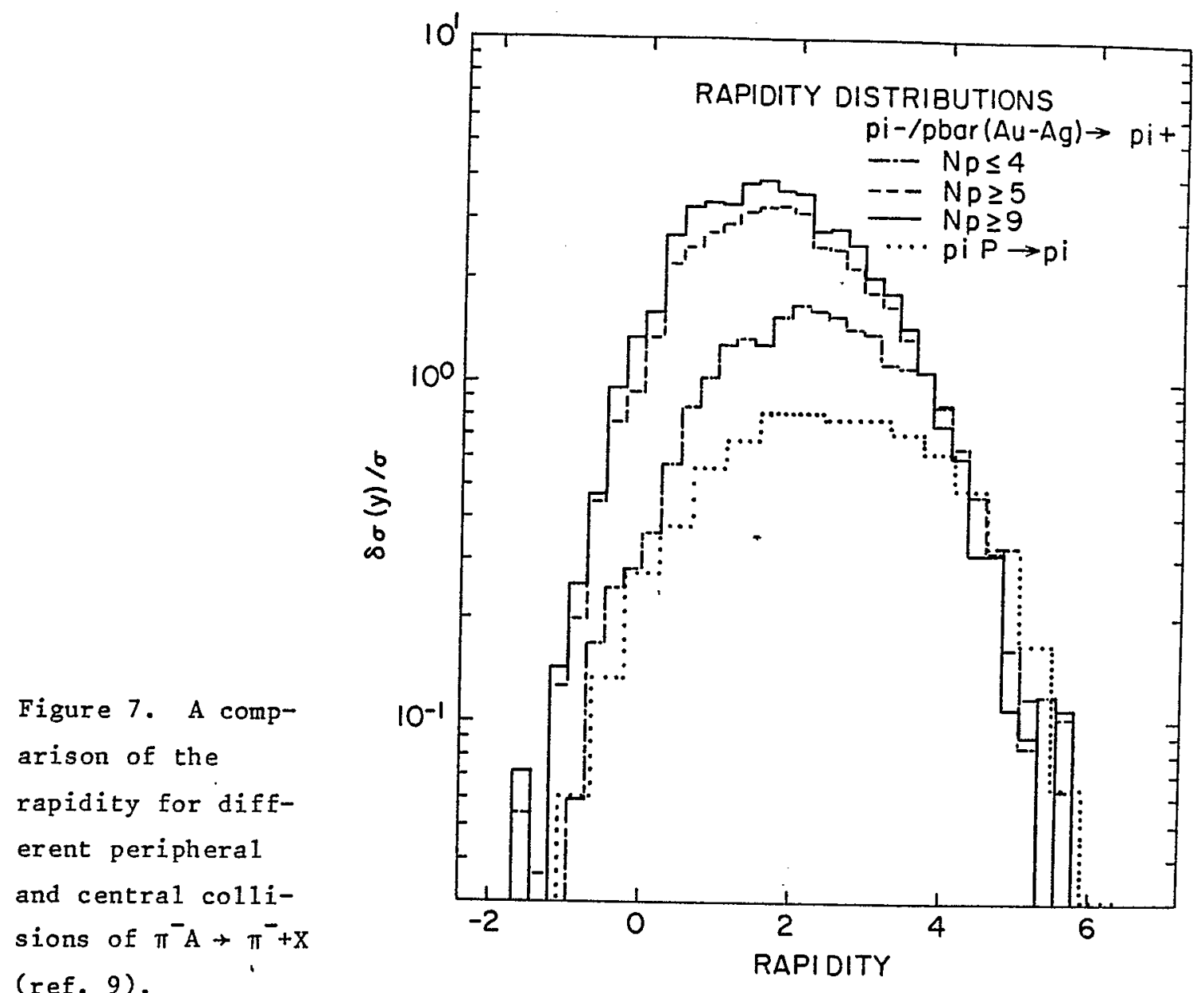

(ref. 9).

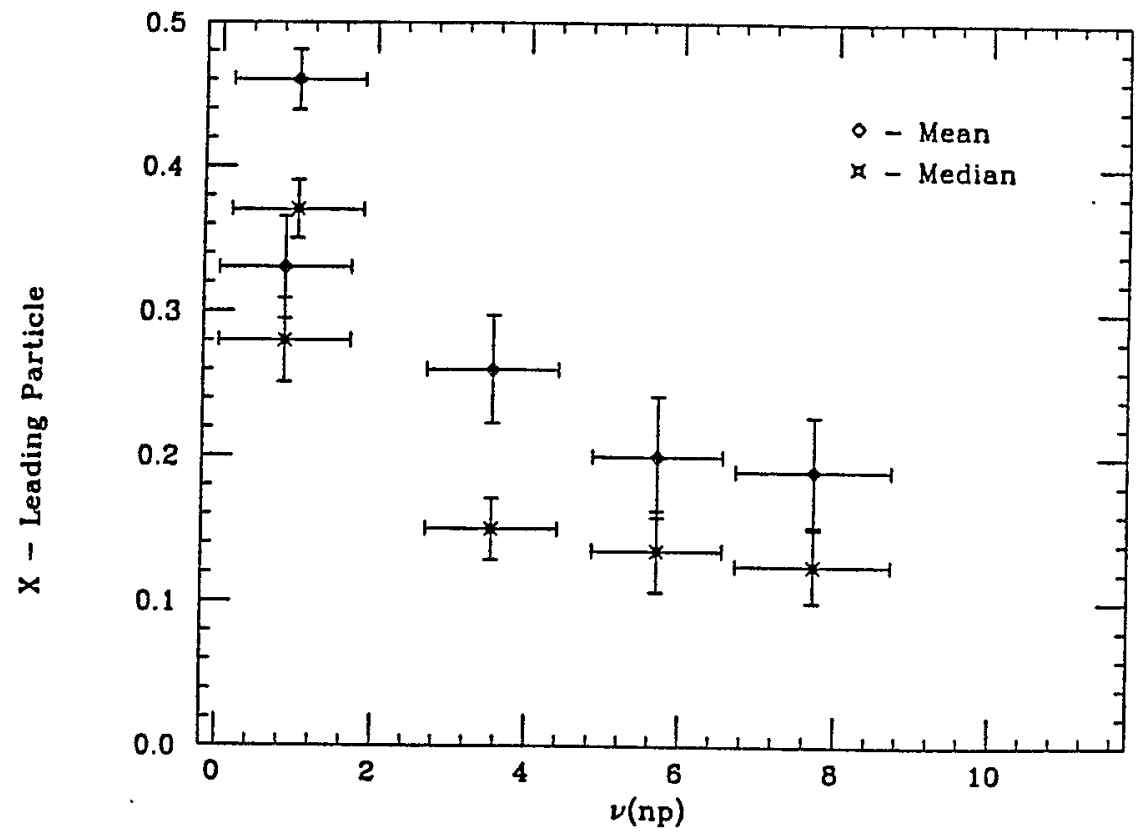

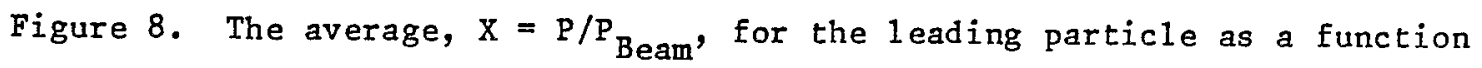
of the number of collisions $\gamma(\mathrm{np})$ from ref. 10. 
we show the total momentum carried by all the particles with $P \geq 10 \mathrm{Gev} / \mathrm{c}$. Here again we see a rapid decrease in the momentum carried by the forward particles with increased interaction number, $\nu$. Figure 10 contains data on the total number of particles and of negative particle in the "projectile fragmentation" region as a function of $\nu$. It is shown that while the momentum or energy carried by the projectile system has changed dramatically as

Figure 9. The total momentum carried by particle with $P \geq$. $\mathrm{Gev} / \mathrm{c}$ as a

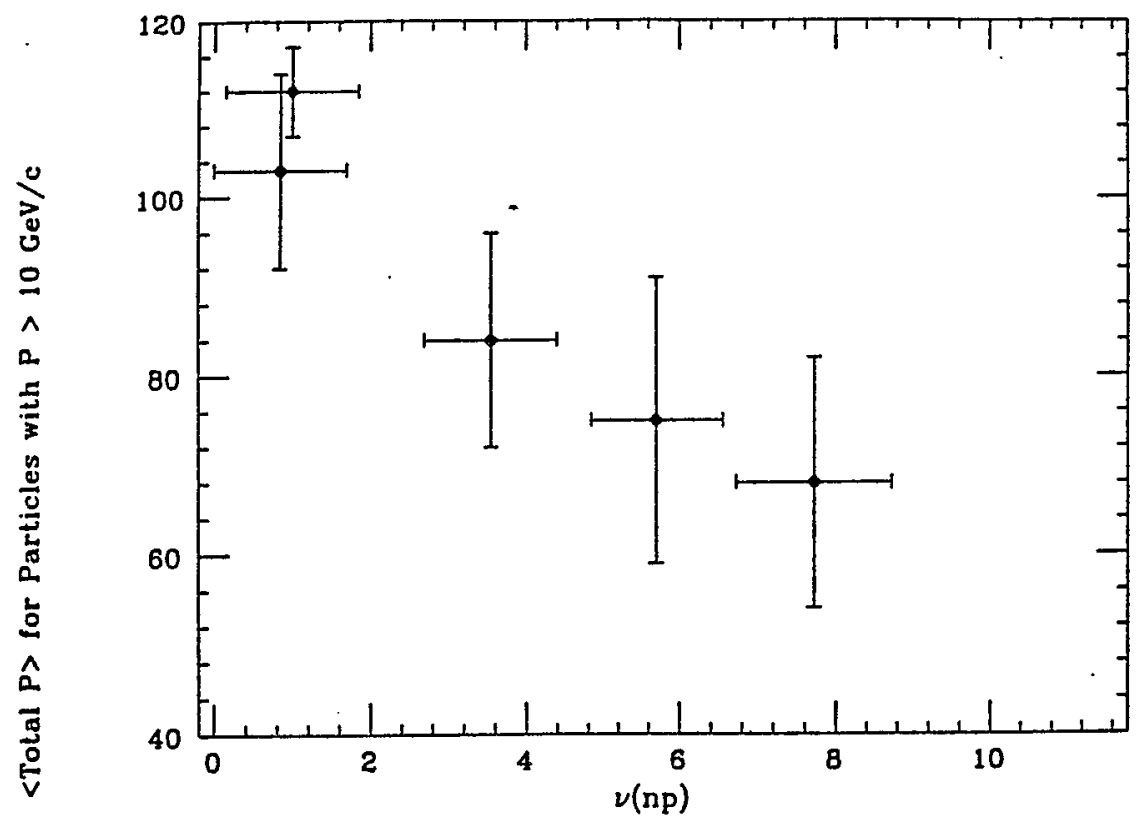

function of $\mathrm{V}$ (ref. 10).

Figure 10.

Total number

of particles and total number of negative particles in the projectile region as a function of $v$ (ref.

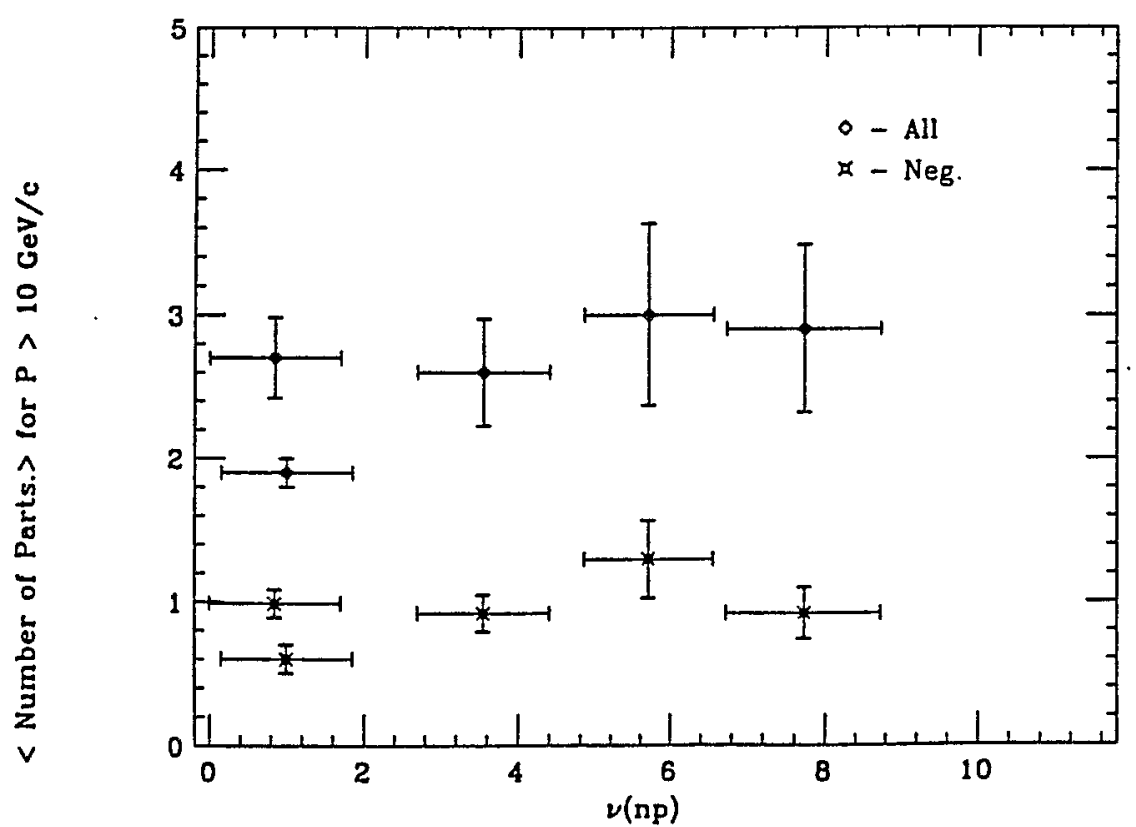
10). 
we increase the number of interactions, the number of particles in this region remains fairly constant. Figure 11 shows the total number of particles and the momenta carried by particles with $P \leq 10 \mathrm{Gev} / \mathrm{c}$. A clear increase is observed in both with increase dv.

The general event properties observed in both experiments are in good agreement. Both experiments complement the inclusive $\mathrm{pA} \rightarrow \mathrm{p}+\mathrm{A}$ data. They measure the average behavior of the leading particles in the fragmentation region as well as the target region.

\section{TRANSVERSE ENERGY DISTRIBUTION IN P-A COLLISION}

Cross sections for inclusive particle production in hadron-nucleus reactions have been found to grow, $\mathrm{d}^{3} \sigma / d \mathrm{p}^{3} \alpha \mathrm{A}^{\alpha}$, such that the exponent $\alpha$ increases from 0.8 at the low $P_{t}$ region to values larger than 1 at $P$ $\mathrm{Gev} / \mathrm{c}$. This effect is known as "anomalous nuclear enhancement." $13-1 \frac{t}{4}$ A similar effect with an even stronger A dependence has been observed when cross sections for production of "jets" are studied. ${ }^{15}$ In this section we want to look at new data on the A dependence of the cross section for large transverse energy, $E_{T}\left(E_{T}=\left.\Sigma\right|_{P_{T}} \mid\right)$, deposited over a limited solid angle. The new data we will look at comes from two experiments, the E609 collaboration at FNAL ${ }^{16}$ and HELIOS/NA34 collaboration at the CERN SPS. ${ }^{17}$ Most data available to date on $E_{T}$ distributions in $\mathrm{PP}$ and $\mathrm{PA}$ collisions were from experiments originally designed to study "jet" production using an "unbiased"

Figure 11.

Total number

of particles and total

momentum energy carried by particles in the backward region as a function of $v$

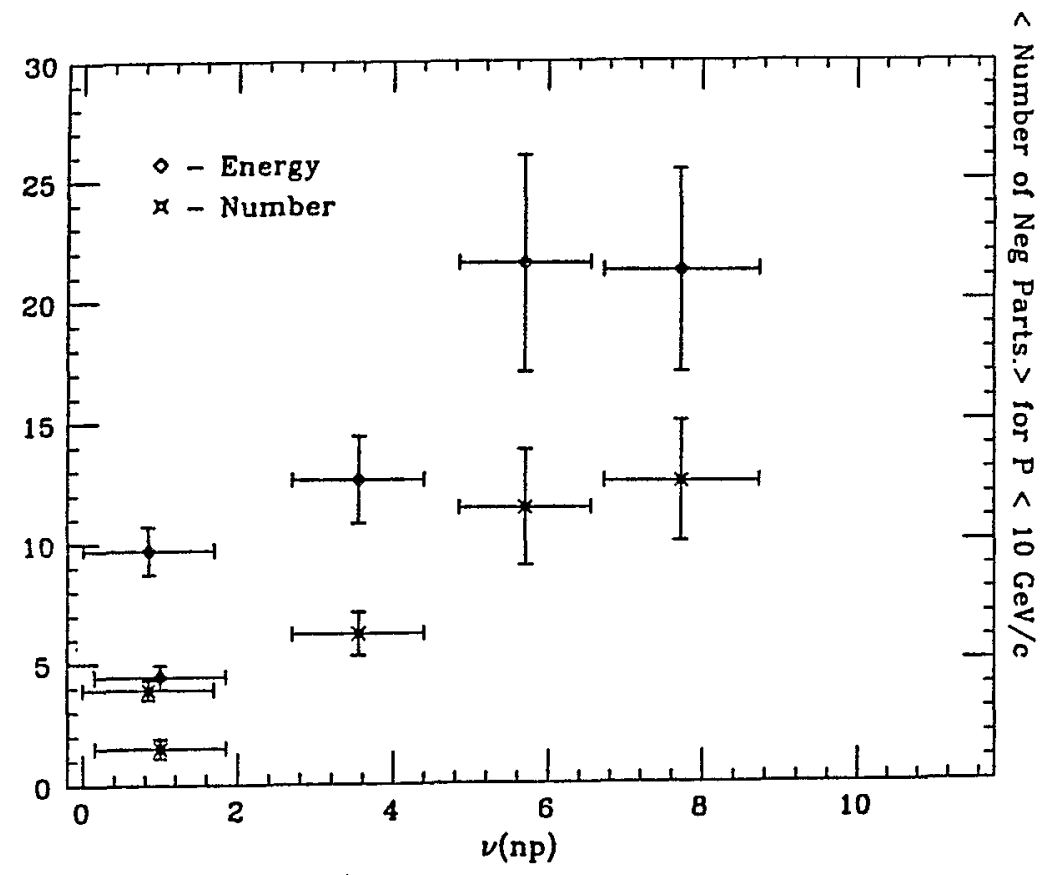

(ref. 10). 
global trigger. ${ }^{18-20}$ The experiments thus were set up to have a $2 \pi-$ calorimeter coverage in $\phi$, and from about -1 to 1 in rapidity as measured in PP center of mass. The results from SPS, ${ }^{19}$ FNAL ${ }^{18}$ and ISR showed that at lower $\sqrt{s} \leq 30 \mathrm{Gev}$, when one triggers on large $\mathrm{E}_{\mathrm{T}}$, the events are dominated by spherical events even at the highest $E_{T}$ bins reached. At higher $\sqrt{s} \geq 45$ Gev, data from the ISR and the $\bar{p}$ p collider at CERN have shown a clear dominance of "jets" if one reaches a sufficiently high $E_{T}$.

Experiment E609 ${ }^{16}$ at FNAL has data triggered on a variety of targets, $\mathrm{H}$, $\mathrm{He}, \mathrm{Be}, \mathrm{C}, \mathrm{Al}, \mathrm{Cu}, \mathrm{Sn}, \mathrm{Pb}$. In figure 12 we show the experimental set up. The calorimeter has a $2 \pi$ coverage in $\phi$ and an acceptance from $30^{\circ}$ to $125^{\circ}$ in $\theta$ in the pp center of mass. Two triggers were used in data taking. One was a global $E_{T}$ trigger where the energy deposited in the calorimeter towers is multiplied by a weight proportional to $\sin \theta$ and then sumed. The other is a "two high trigger" where two towers are required to have energy greater than a given threshold. This trigger tends to pick out events that have a "jet" structure. In figure 13 we show the differential cross section $\mathrm{dE} / \mathrm{dE}_{T}$ vs $E_{T}$ for the different targets. Note that the data stops at $E_{T}$ of about 20 Gev, which is just below the pp kinematic limit. The cross sections of the heavier targets are still very large at that $E_{T}$, which is well below the $\mathrm{pA}$ kinematic limit. Note also that the slope of the distribution becomes flatter as the $A_{1}$ of the target increases. In figure 14 we show the relative cross section $\sigma(\mathrm{pA}) / \sigma(\mathrm{A} * \sigma(\mathrm{pp}))$ v.s $A$ for different bins in $E_{\mathrm{T}}$. One sees clearly that as $E_{T}$ increases the $A$ dependence becomes much steeper.

In figure 15 we show the planarity distribution for the global and two high trigger events. Note that "jet" events, which are assumed to arise from a single hard constituent scattering will appear at planarity near one, while spherical events, which 'could be a product of a large number of soft interactions, will appear at lower planarity. Figure 16 shows the relative cross section $\sigma(\mathrm{pA}) / \sigma(\mathrm{A} * \sigma(\mathrm{pp}))$ vs A for different planarity bins. It is clear that there is a strong dependence of the cross section of $A$ in all the planarity bins but that the dependence is much stronger for spherical events $(P \ll 1)$.

The Helios ${ }^{17}$ data were taken in 1984. The collaboration took only $P \mathrm{~Pb}$ data at $200 \mathrm{Gev} / \mathrm{c}$. In figure 17 we show the experimental setup. Note that this calorimeter has $2 \pi$ acceptance in $\phi$ and a large $n$-pseudo rapidity acceptance, from $\eta 0.6$ to 2.4. Thus it does not restrict itself to the central rapidity region in the pp center of mass but covers in addition the more backward region where one would expect to find more of the energy in central $\mathrm{pA}$ collisions. In figure 18 we show the preliminary $\mathrm{E}_{\mathrm{T}}$ distribution corrected for effects like energy resolution in the calorimeter, finite 


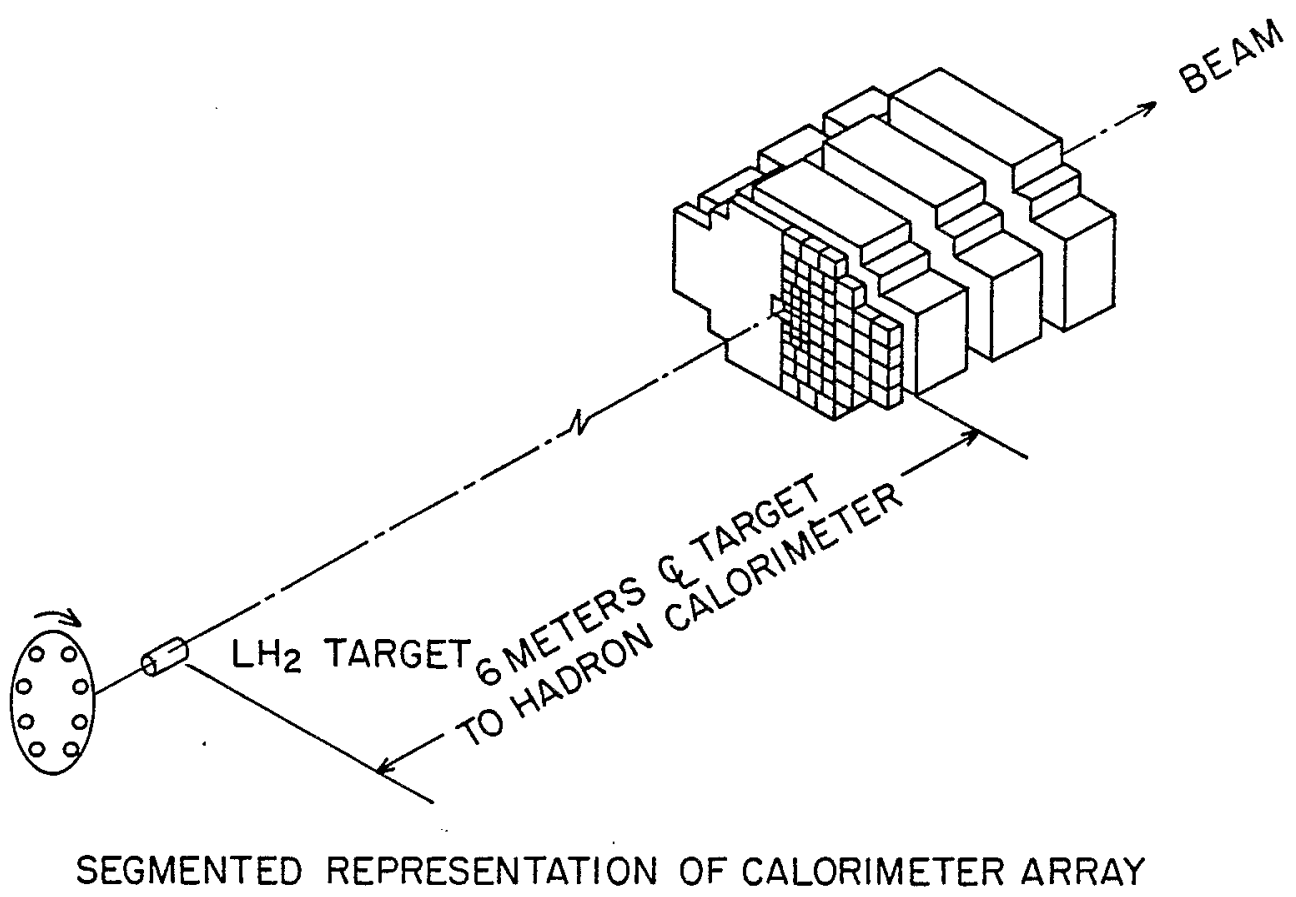

Figure 12. Experimental setup for experiment. E609 (ref. 16).

Figure 13. The differention $E_{T}$ cross section for global trigger, $d \sigma / d E_{T}$ vs $E_{T}$ from ref. 16.

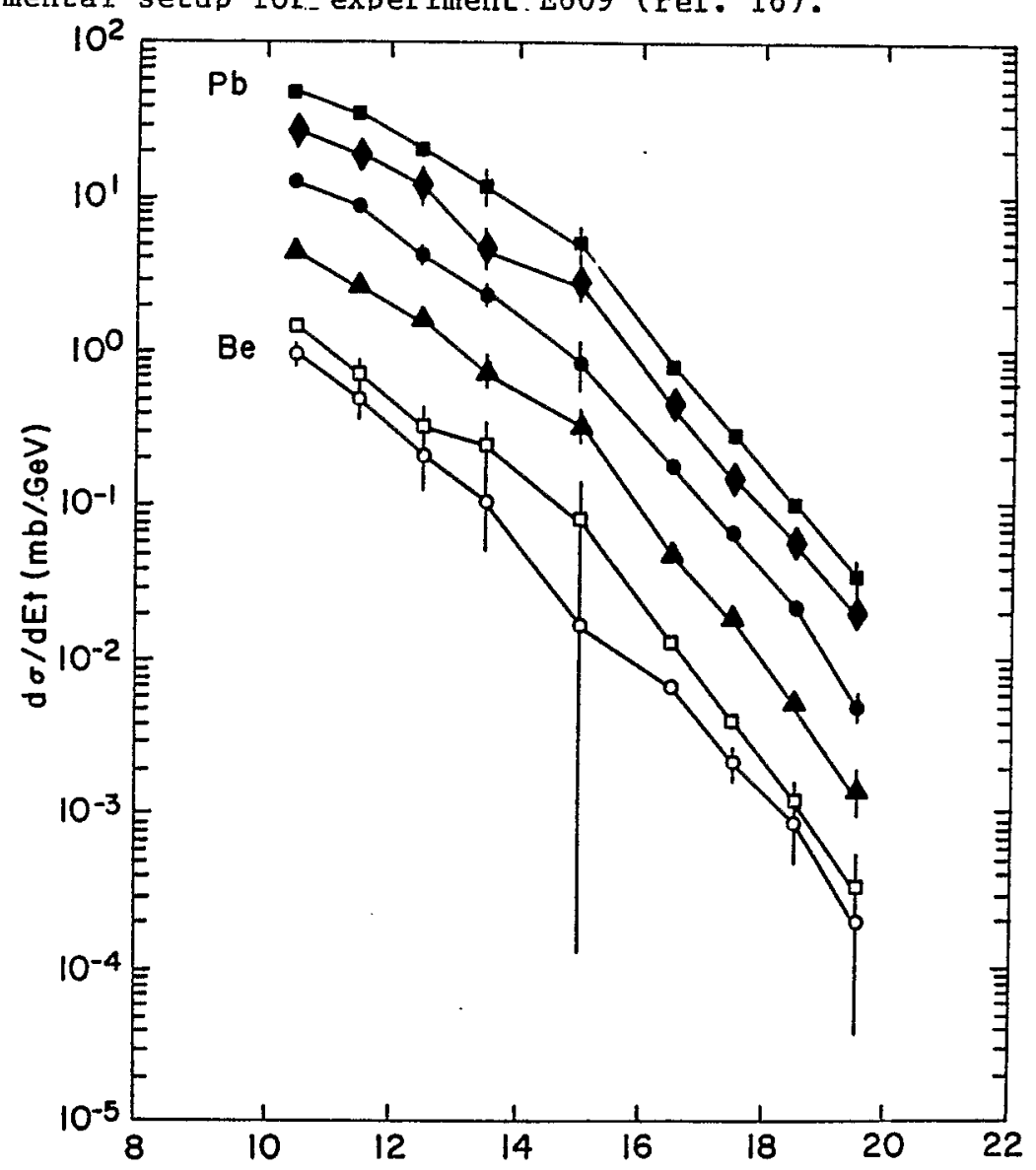




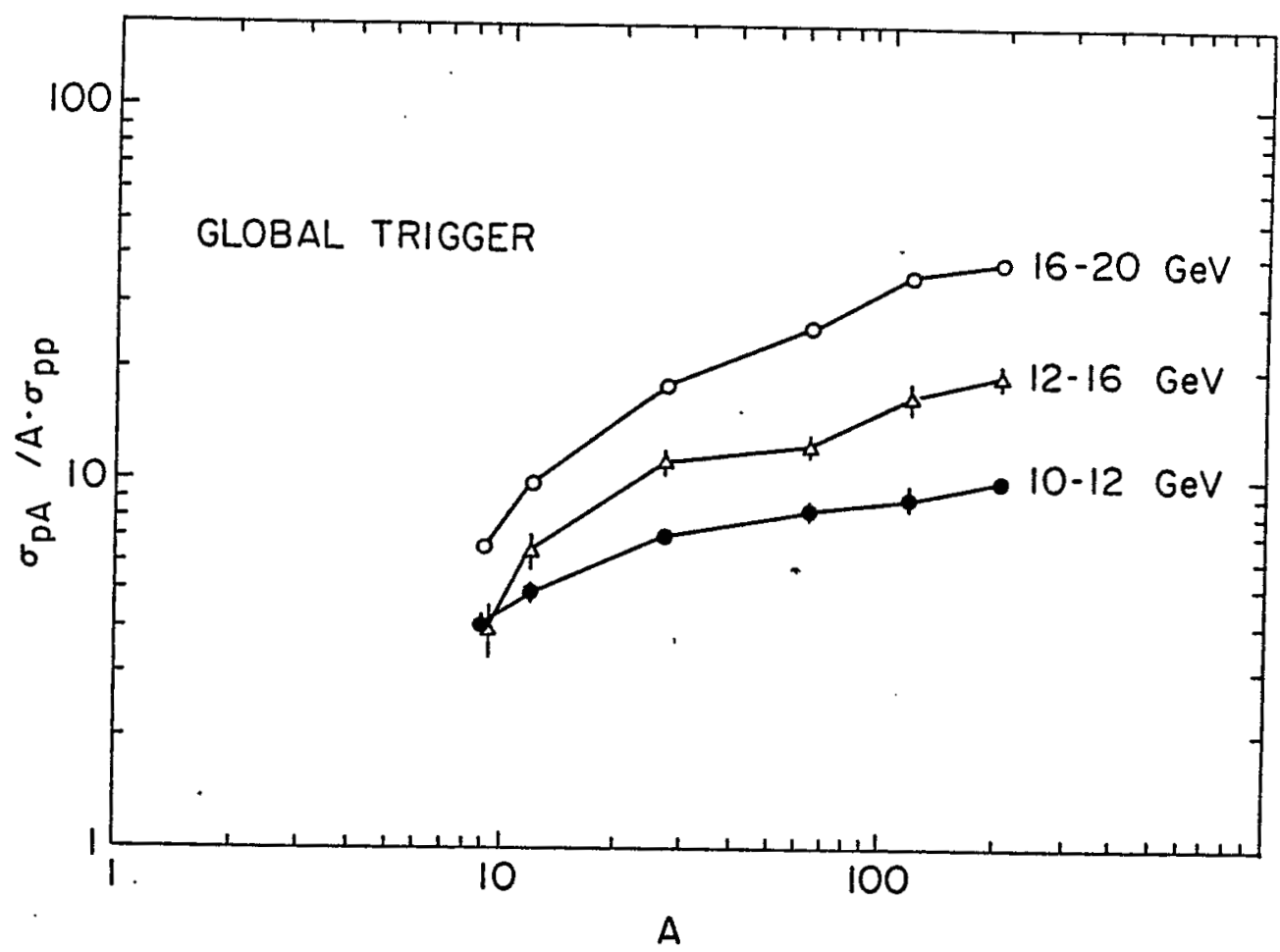

Figure 14. Relative $\mathrm{PA}$ cross section to $\mathrm{A}^{*} \mathrm{pp}$ cross section vs A (ref. 16).

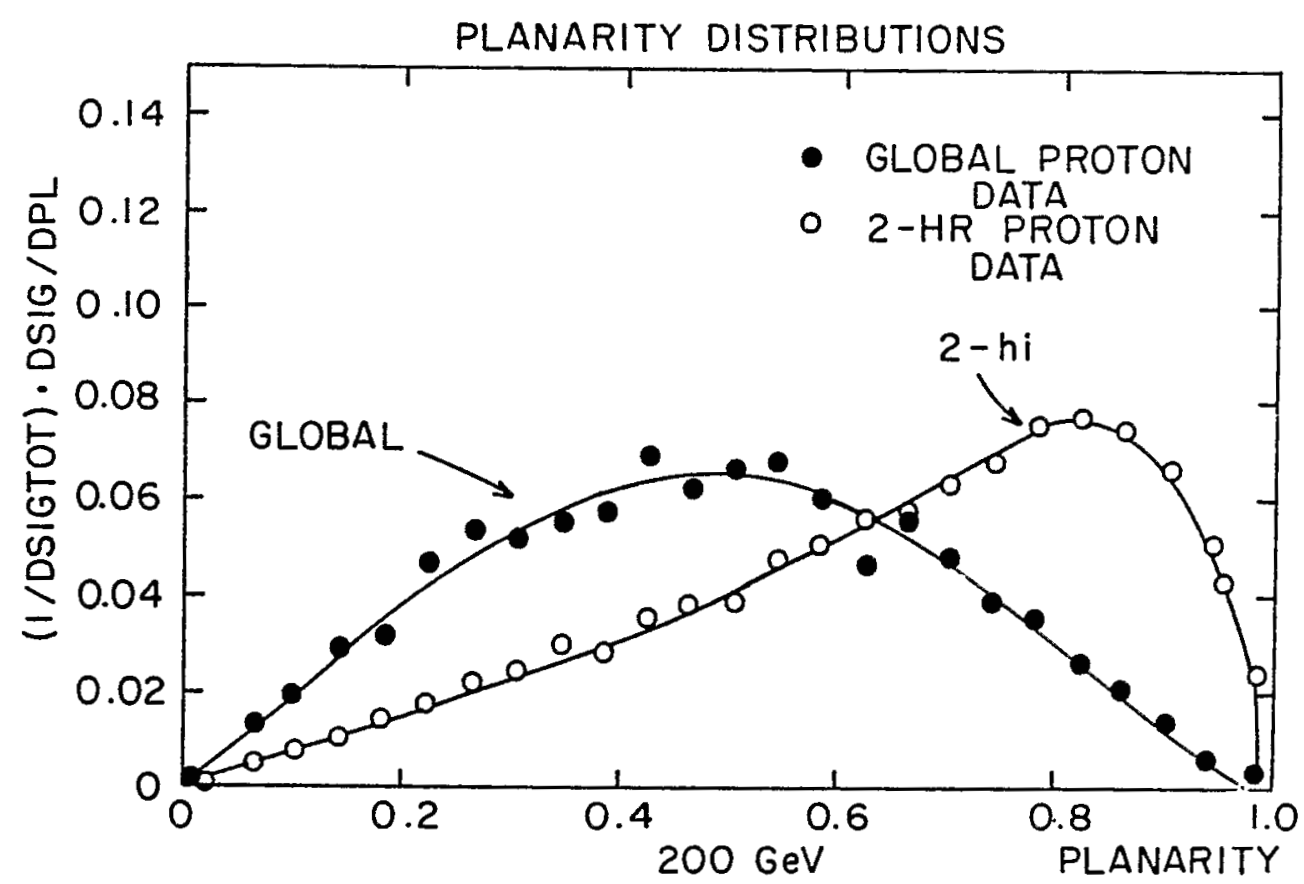

Figure 15. Planarity distribution for global and two high trigger events (ref. 16). 


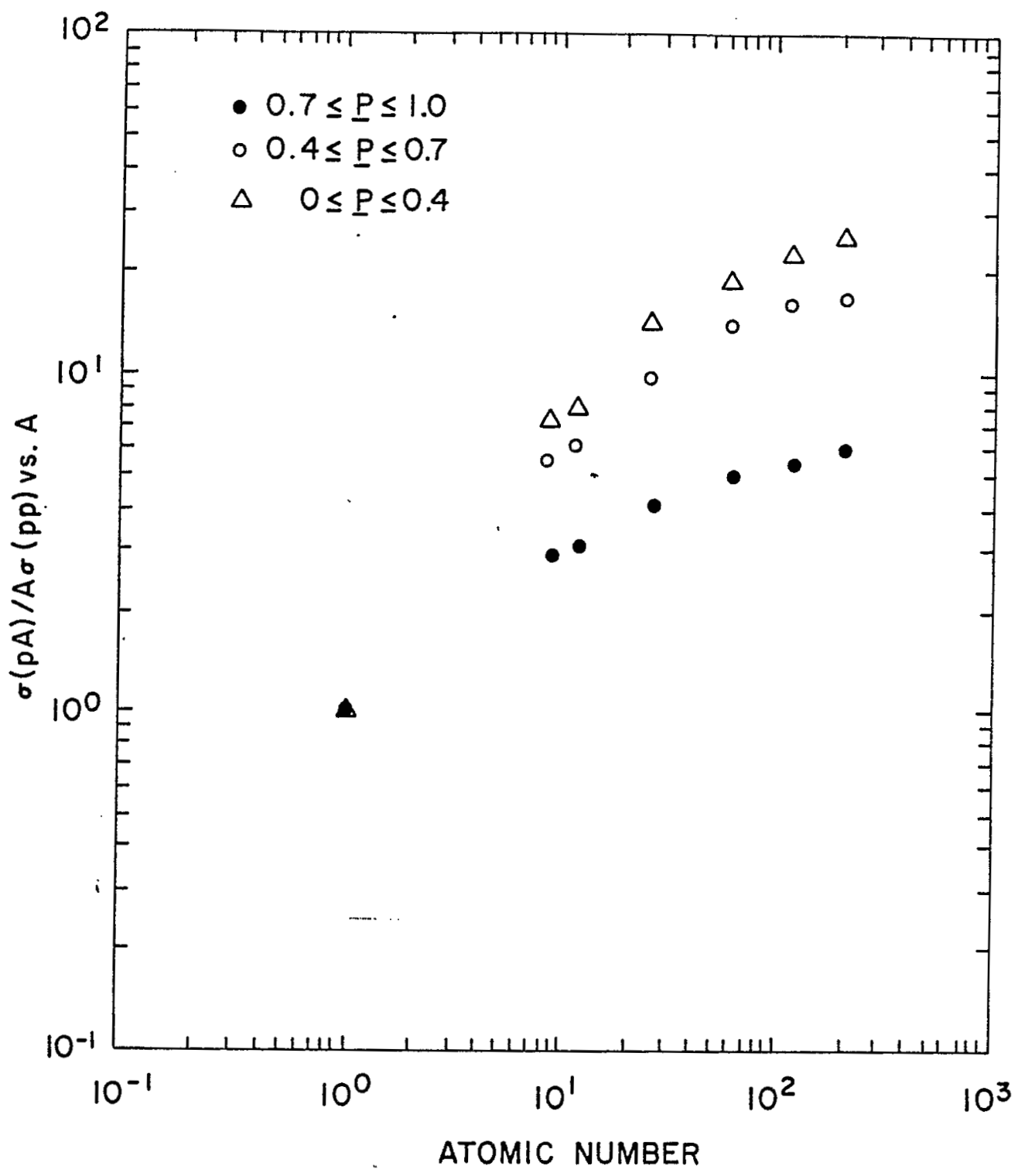

Figure 16. Relative $\mathrm{PA}$ cross section to $\mathrm{A}^{*} \mathrm{PP}$ cross section vs A for different planarity bins.

calorimeter granularity, finite shower size and others. Note that the distribution goes well beyond the pp kinematic limit. The corrections are fairly large and the dotted lines give a measure of the uncertainty in the unfolding procedure. This result is still preliminary, but it is clear that the distribution shows a remarkably large cross section well beyond the pp kinematic limit.

In figure 20 we show the energy flow as a function of pseudo rapidity, $\eta$, for the different $E_{T}$ bins. The character of the distribution shows. a dramatic change: while at lower $\mathrm{E}_{\mathrm{T}}$ it is strongly forward peaked, 
at higher $E_{T}$ it flattens out with some suggestion of being even backward peaked. In figure 21 we show the $\langle\eta\rangle$ as a function of $E_{T^{*}}$. A rapid decrease in $\langle n\rangle$ is seen with increased $E_{T}$.

There have been a number of attempts to explain this data using Hijet, and Lund Monte Carlo as well as by wounded nucleon model. Until now some models have succeeded in explaining the data up to $E_{T} \simeq 20 \mathrm{Gev}$ but have failed at the higher $E_{T}$, understimating the data by at least an order of magnitude. The large cross sections at high $\mathrm{E}_{\mathrm{T}}$ over limited rapidity region translate into large energy density, a very encouraging result for expected densities in AA collisions.

\section{CONCLUSTONS}

We have concentrated our survey of hadron-nucleus collisions on new data which became available lately. The inclusive $\mathrm{pp} \rightarrow \mathrm{p}+\mathrm{X}$ measurements have been shown to be of fundamental importance to our understanding of stopping power; unfortunately the new data has not resolved some of the problems with existing data but has raised new questions about the different data sets which are now clearly inconsistent. More data is clearly needed to resolve this,

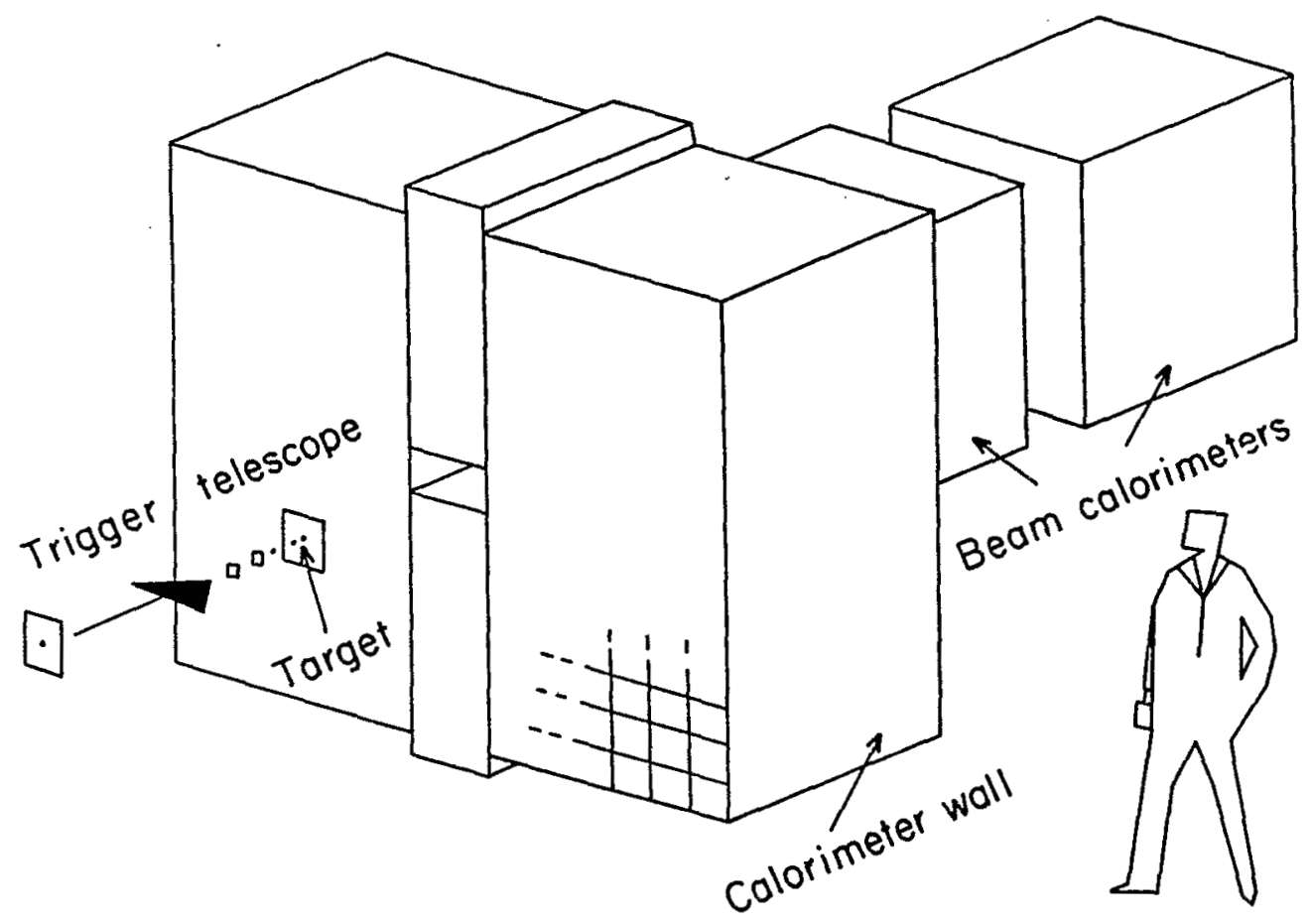


The bubble chamber data are limited in statistics but offer a good look at the global properties of the events.

(i) Leading particles of peripheral pA collisions and p-light ion collisions are similar.

(ii) Leading particles are strongly absorbed in central collisions.

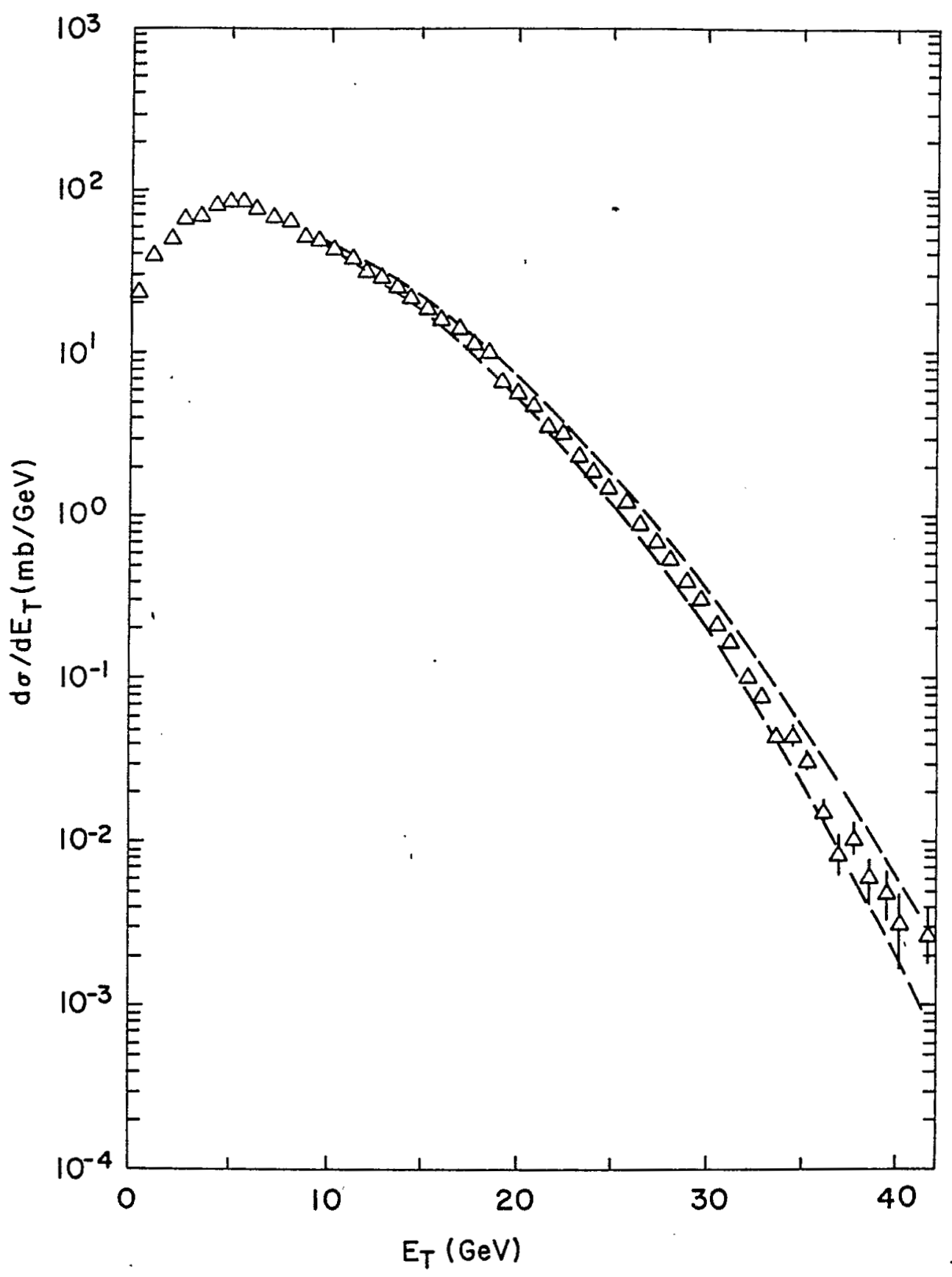

Figure 18. $E_{T}$ distribution for PPb collisions (ref. 17). 
Figure 19. Energy flow vs pseudo rapidity for different $\mathrm{E}_{\mathrm{T}}$ bins (ref. 17).

Figure 20. Average pseudo rapidity, <n> vs $\mathrm{E}_{\mathrm{T}}$ from ref. 17 .
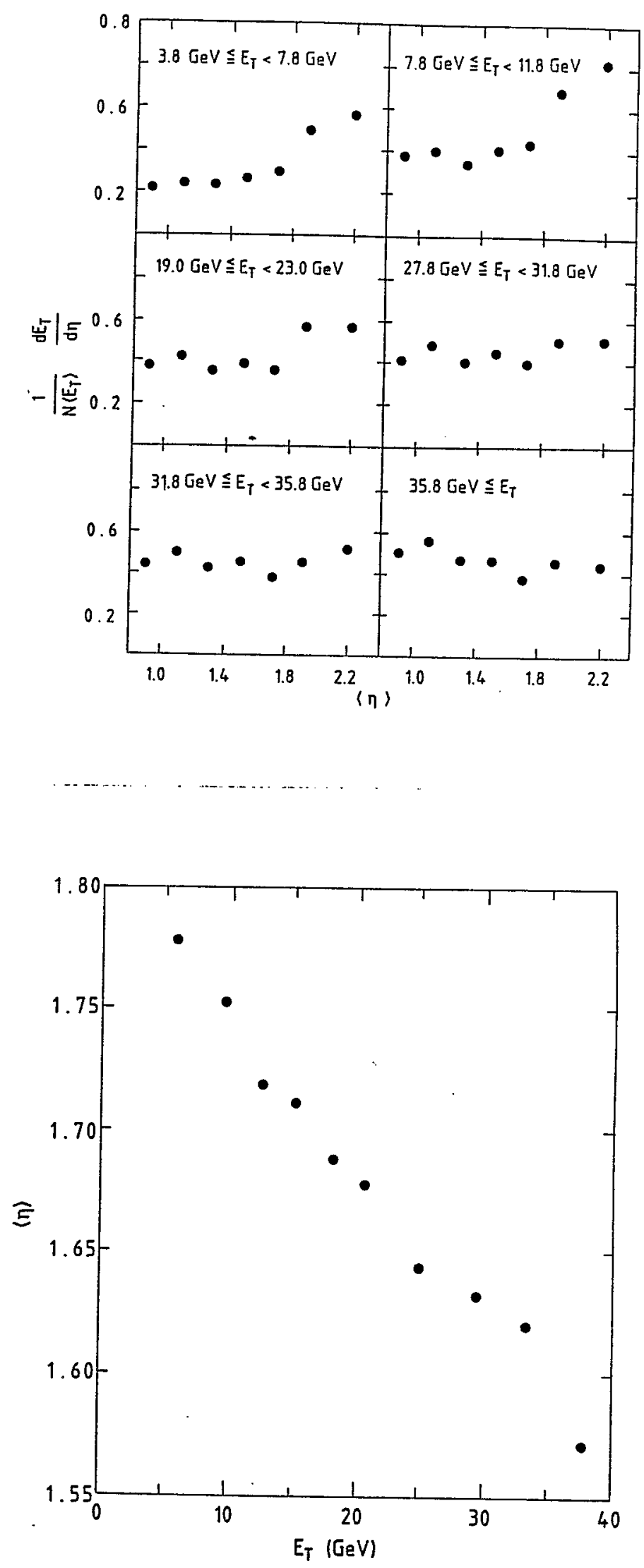
(iii) KNO scaling gives a fair representátion of the negative pion distribution.

(iv) Energy is transferred from the leading particle and is deposited in the background region at a rate of about $3 \mathrm{Gev} /$ Fermi.

The high $E_{T}$ data allow investigating the rare events where a large amount of energy is deposited in a given rapidity region. The data shows a large cross section for high $E_{T}$ rising much faster than $A$, The A dependence of the spherical events $(P \ll 1)$ is greater than that of jet events. The $\langle\eta\rangle$ rapidity shows a marked decrease with increasing $E_{T}$, as do the energy flow $\mathrm{DE}_{\mathrm{T}} / \mathrm{d}$ distribution. No model to date was able to explain the large cross section at high $\mathrm{E}_{\mathrm{T}}$. This effect is encouraging as it implies with high probability that one can reach high densities in AA collisions.

\section{REFERENCES}

1) W. Busza and A. Goldhaber, Phys. Lett. 139B (1984) 235; W. Busza, Nuc1. Phys. A418 (1984) 635c.

2) D.S. Barton et a1., Phys. Rev. D2

3) S. Daté, M. Gyulassy and H. Sumiyoshi, Phys. Rev. D32 (1985) 619.

4) R.C. Hwa, Phys. Rev. Lett. 52 (1984) 492.

5) C. Wong, Phys. Rev. Lett. 52 (1984) 1393; J. Hufner and A. Klar, Phys. Lett. 14 B B i91984) 167 .

6) R. Bailey, Z. Particles and Fields, Z. Phys. C. 29 (1985) 1.

7) T. Eichten et a1., Nucl. Phys. B44 (1978) 333.

8) L.P. Remsberg et al., Invited talk, "7th High Energy Heavy Ion Study," BNL preprint.

9) W.D. Walker et a1., private communication.

10) R.J. Ledoux et al., talk presented at International Workshop XIV, "Gross Properties of Nuclei and Nuclear Excitation, Hirschegg Austria, 1986, and private communication.

11) W.Q. Chao et a1., Nucl. Phys. A395, (1983) 482.

12) C. De Marzo et al., Phys. Rev. D29 (1984) 2476.

13) J.W. Cronin et a1., Phys. Rev. D11 (1975) 3105.

14) T. Akesson et a1., Nuc1. Phys. B246. (1984) 408.

15) C. Bromberg et al., Phys. Rev. Lett. 42 (1979) 1202.

16) H.E. Miettinen et al., E609-collaboration, private communication.

17) T. Akesson et al., Helios collaboration. CERN-EP, private communication. 
18) B. Brown et a1., Phys. Rev. Lett. 50 (1983) 11.

19) C. De Marzo et al., Phys. Lett. 112B (1982) 173.

20) T. Akesson et al., Phys. Lett. 128 (1983) 354.

21) T. Ludlam, et al., Hijet event generator, these proceedings.

22) B. Anderson, et a1., LUTP 86-3, Univ. of Lund, Sweden, February 1986, and these proceedings. 